\title{
New Reagents for Increasing ESI Multiple Charging of Proteins and Protein Complexes
}

\author{
Shirley H. Lomeli, ${ }^{a}$ Ivory X. Peng, ${ }^{\text {a }}$ Sheng Yin, ${ }^{a}$ \\ Rachel R. Ogorzalek Loo, ${ }^{b}$ and Joseph A. Loo ${ }^{a, b}$ \\ ${ }^{a}$ Department of Chemistry and Biochemistry, David Geffen School of Medicine, University of California-Los \\ Angeles, Los Angeles, California, USA \\ ${ }^{\mathrm{b}}$ Department of Biological Chemistry, David Geffen School of Medicine, University of California-Los \\ Angeles, Los Angeles, California, USA
}

\begin{abstract}
The addition of $m$-nitrobenzyl alcohol (m-NBA) was shown previously (Lomeli et al., J. Am. Soc. Mass Spectrom. 2009, 20, 593-596) to enhance multiple charging of native proteins and noncovalent protein complexes in electrospray ionization (ESI) mass spectra. Additional new reagents have been found to "supercharge" proteins from nondenaturing solutions; several of these reagents are shown to be more effective than $m$-NBA for increasing positive charging. Using the myoglobin protein-protoporphyrin IX (heme) complex, the following reagents were shown to increase ESI charging: benzyl alcohol, $m$-nitroacetophenone, $m$-nitrobenzonitrile, $o$-NBA, $m$-NBA, $p$-NBA, $m$-nitrophenyl ethanol, sulfolane (tetramethylene sulfone), and $m$-(trifluoromethyl)-benzyl alcohol. Based on average charge state, sulfolane displayed a greater charge increase $(61 \%)$ than $m$-NBA $(21 \%)$ for myoglobin in aqueous solutions. The reagents that promote higher ESI charging appear to have low solution-phase basicities and relatively low gas-phase basicities, and are less volatile than water. Another feature of mass spectra from some of the active reagents is that adducts are present on higher charge states, suggesting that a mechanism by which proteins acquire additional charge involves direct interaction with the reagent, in addition to other factors such as surface tension and protein denaturation. (J Am Soc Mass Spectrom 2010, 21, 127-131) @ 2010 American Society for Mass Spectrometry
\end{abstract}

$\mathrm{E}$ lectrospray ionization is distinguished among other desorption/ionization sources for mass spectrometry by its ready generation of multiply charged molecules, the value of which was recognized early by Fenn's group: "This feature is very attractive in that it extends the effective mass range of any analyzer by a factor equal to the number of charges per ion. Moreover, because the ions have lower $\mathrm{m} / \mathrm{z}$ values, they are generally easier to detect and weigh than are singly charged ions of the same mass [1]."

After the utility of ESI-MS for protein analysis was demonstrated, multiple charging was rapidly extended to tandem mass spectrometry of large peptides and proteins $[2,3]$. Dissociation efficiency of large molecules is enhanced with increasing charge, allowing sequenceinformative product ions to be measured. Early ESI-MS studies investigated the potential of different solvents to increase charging [4]. However, useful enhancement of ESI multiple charging was not described clearly until Iavarone and Williams reported "supercharging" promoted by agents such as $m$-nitrobenzyl alcohol ( $m$-NBA) $[5,6]$. For protein analyses, their work largely investigated solutions considered to be denaturing.

Address reprint requests to Dr. R. R. Ogorzalek Loo and Dr. J. A. Loo, University of California-Los Angeles, Molecular Biology Institute, Los Angeles, CA 90095, USA. E-mail: RLoo@mednet.ucla.edu and E-mail: JLoo@chem.ucla.edu
Recently, we demonstrated that multiple charging of noncovalent protein complexes could be increased in ESI-MS when spectra are obtained from nondenaturing protein solutions containing up to $1 \%(\mathrm{vol} / \mathrm{vol}) m$-NBA [7]. Increases in charge ranged from $8 \%$ for the $690 \mathrm{kDa}$ $20 \mathrm{~S}$ proteasome complex to $48 \%$ additional charge for zinc-bound $29 \mathrm{kDa}$ carbonic anhydrase protein. These protein structures were considered native because no dissociation of noncovalently bound ligands, subunits, or metal ions was observed from complexes sprayed out of the solution compositions described, nor were differences revealed by circular dichroism or hydrogen/deuterium exchange solution phase measurements.

The present report describes new compounds as potent as $m$-NBA in supercharging native protein complexes (e.g., myoglobin), and provides preliminary data revealing their utility with denaturing solutions. Many of the newly described compounds for increasing ESI multiple charging are structurally similar to $m$-NBA. We discuss possible physical and chemical properties impacting multiple charging behavior.

\section{Experimental}

Positive ion ESI mass spectra were acquired in similar fashion to our previous study [7] and with two different systems: a hybrid quadrupole time-of-flight (QTOF) 
mass spectrometer with a Triwave ion mobility (IM) separator (QTOF/IM; Waters Synapt HDMS, Manchester, UK) and an LTQ-FT Ultra Fourier transform mass spectrometer (Thermo Fischer Scientific, San Jose, CA, USA). The nanoESI source using borosilicate glass capillaries with $\mathrm{Au} / \mathrm{Pd}$ coatings (Proxeon Biosystems, Odense, Denmark) was operated at low analyte flow conditions (50 $\mathrm{nL} / \mathrm{min})$. All protein samples were desalted with $20 \mathrm{mM}$ ammonium acetate before analysis using centrifugal filter devices. The final protein concentration for ESI-MS measurements was $\sim 5 \mu \mathrm{M}$ in $20 \mathrm{mM}$ ammonium acetate, $\mathrm{pH}$ 6.8. Increased ESI-generated charge states for native protein complexes were enabled by adding $m$-NBA and other compounds directly to the protein solutions. ESI mass spectra of proteins in denaturing solutions were acquired with an LTQ linear ion trap system (Thermo Fischer Scientific) with a nanoESI source (New Objective, Woburn, MA, USA).

For a given comparison, instrument settings were held constant as reagent concentration was varied, to ensure that changes to the stability and charging of protein complexes may properly be attributed to changes in solution conditions.

Equine myoglobin, bovine insulin, and phosphorylase $b$ (glycogen phosphorylase, rabbit muscle) were purchased from Sigma-Aldrich (St. Louis, MO, USA), as were ortho-, meta-, and para-nitrobenzyl alcohol, benzyl alcohol, $\alpha$-(4-pyridyl $N$-oxide)- $N$-tert-butylnitrone (POBN), 3-nitrophenyl ethanol, nitrobenzene, 3-nitroacetophenone, 3-nitrobenzonitrile, 3-nitrophenol, sulfolane, and 3trifluoromethyl benzyl alcohol.

\section{Results and Discussion}

Supercharging, the increased ESI charging observed when $m$-NBA and other liquids are added to denatured protein solutions, has been attributed to the charged residue model (CRM) relationship linking the Rayleigh limit of droplet charge $\left(z_{R}\right)$ to surface tension $(\gamma)$, droplet radius $(R)$, the permittivity of vacuum $\left(\varepsilon_{0}\right)$, and elementary charge, $e$ [8]:

$$
z_{R} e=8 \pi\left(\varepsilon_{0} \gamma \mathrm{R}^{3}\right)^{1 / 2}
$$

Mature droplet surface tension is often approximated by the value for the lowest-vapor pressure solvent component $[6,8]$.

Inconsistencies with this supercharging model $[5,6]$ have been noted: (1) the CRM is suggested to apply only to nondenatured proteins [4], (2) that mature droplet composition should reflect solvent evaporation rates rather than equilibrium vapor pressures, questions some experimental support [9], and (3) folded protein charge state distributions from $\mathrm{pH} 7$ aqueous solutions did not shift to lower charge following addition of $40 \%$ propylene glycol [9]. Our results for $m$-NBA addition [7], displaying increased charging with littleto-no dissociation of noncovalently bound ligands/ subunits from aqueous, near neutral-pH solutions are also inconsistent with this model. Although $m$-NBA has a reported surface tension of $50 \pm 5 \mathrm{mN} / \mathrm{m}$ [10], water's value at $25^{\circ} \mathrm{C}$ is higher, $72.0 \mathrm{mN} / \mathrm{m}$ [11].

Attempting to locate new supercharging agents with $m$-NBA-like ability to increase charging from nearnative solutions, we examined analogous molecules, employing the myoglobin-heme complex as the primary test protein. Numerous reagents were examined at concentrations up to their maximum solubility, or until protein denaturation was evident (as judged by significant loss of noncovalently-associated heme or by a discontinuous change in charge profile with increasing reagent concentration).

Nitrobenzyl alcohol's ortho- and para-isomers, solids at room temperature, and the liquid meta-isomer, are all potent charge enhancing reagents. When electrosprayed from nondenaturing solutions, holo-myoglobin displayed 7+-9+ heme-associated ions. Addition of $o$-NBA to $20 \mathrm{mM}$ increased the level of protonation without detaching heme, elevating the most intense ion's charge state by almost two units. The para-isomer, $p$-NBA, also increased the charge of myoglobin cations without dislodging heme, up to the $p$-NBA solubility limit of $12 \mathrm{mM}$, at which concentration the most abundant protein charge state was one unit higher. Previously identified supercharging agent $m$-NBA increased holo-myoglobin's average charge by almost two units when present at $40 \mathrm{mM}$ [7].

Extending the alkyl alcohol chain by a $-\mathrm{CH}_{2}$ unit to form m-nitrophenyl ethanol yielded a reagent also affording supercharging. Other effective nitrobenzene derivatives included $m$-nitroacetophenone and $m$ nitrobenzonitrile. Despite its limited solubility in the ammonium acetate/water solvent $(<13 \mathrm{mM})$, $m$ nitroacetophenone increased holo-myoglobin average charge by almost two units and the maximum charge increased from $9+$ to $12+$. Similarly, addition of $1.5 \mathrm{mM}$ $m$-nitrobenzonitrile increased the maximum charge state for holo-myoglobin to $15+$. Thus, each of the reagents tested elevates charge to a different extent (Table 1, Figure 1).

Among the reagents that promote more multiple charging than the NBAs is sulfolane (tetramethylene sulfone). For example, addition of $276 \mathrm{mM}$ sulfolane increased the maximum charge state of holo-myoglobin from $9+$ to $18+$ (Figure 1 ); maximum charging for holo-myoglobin with $m$-NBA is only $12+$. The average charge state of holo-myoglobin increased with increasing sulfolane concentration (Supplemental Materials, which can be found in the electronic version of this article). Sulfolane was used previously as a liquid matrix in the early days of fast atom bombardment (FAB) $[12,13]$, and more recently for infrared matrixassisted laser desorption/ionization (IR-MALDI) [14]. (Coincidentally, m-NBA was used as a FAB matrix [15] and as a liquid matrix for UV-MALDI [16].) ESI-MS behavior of other protein-ligand and protein-protein complexes, such as $29 \mathrm{kDa}$ carbonic anhydrase-Zn, 22 $\mathrm{kDa}$ adenylate kinase-ATP, and the $195 \mathrm{kDa}$ phosphor- 
Table 1. Compounds that enhance ESI-MS charging of myoglobin noncovalent complex

\begin{tabular}{|c|c|c|c|c|}
\hline Compound & Structure & Average charge ${ }^{a}$ & Maximum charge & Concentration $^{\mathrm{b}}$ \\
\hline None & - & 8.2 & 9 & - \\
\hline Benzyl alcohol & & 9.5 & 14 & $240 \mathrm{mM}$ \\
\hline$m$-Nitroacetophenone & & 9.4 & 13 & $\sim 10 \mathrm{mM}$ \\
\hline$m$-Nitrobenzonitrile & & 9.8 & 15 & $1.5 \mathrm{mM}$ \\
\hline o-Nitrobenzyl alcohol & & 10.5 & 14 & $39 \mathrm{mM}$ \\
\hline$m$-Nitrobenzyl alcohol & & 9.9 & 12 & $40 \mathrm{mM}$ \\
\hline$p$-Nitrobenzyl alcohol & & 9.1 & 11 & $12 \mathrm{mM}$ \\
\hline$m$-Nitrophenyl ethanol & & 11.0 & 17 & $23 \mathrm{mM}$ \\
\hline Sulfolane & & 13.2 & 18 & $276 \mathrm{mM}$ \\
\hline$m$-(Trifluoromethyl)-benzyl alcohol & & 8.7 & 10 & $66 \mathrm{mM}$ \\
\hline
\end{tabular}

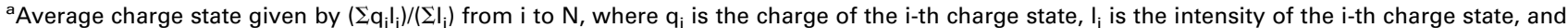
$\mathrm{N}$ is the number of measured charge states in a mass spectrum.

${ }^{b}$ Maximum concentration of compound tested that yielded increasing charge, limited by solubility, heme-loss, or a discontinuous change to the holo-myoglobin charge state distribution.

ylase $b$ dimer, were tested with some of the reagents above that increased charging for holo-myoglobin (Supplemental Materials); in all cases, the noncovalent complex's charging increased without significant subunit or ligand dissociation.

In contrast, many related compounds did not promote increased charging, such as nitrobenzene and $m$-nitrophenol. Nitrobenzene additions to $0.5 \% \mathrm{vol} / \mathrm{vol}$ did not alter myoglobin charge state distributions. At $0.9 \% \mathrm{vol} / \mathrm{vol}(88 \mathrm{mM})$, close to the miscibility limit, unresolved peaks tailed to higher $m / z$, suggesting adduct binding and/or incomplete desolvation. Adding 5 $\mathrm{mM} \alpha$-(4-pyridyl $N$-oxide)-N-tert-butylnitrone (POBN) reduced charging, shifting the most intense holomyoglobin ion from $8+$ to $7+$.

Several of the compounds tested appeared to induce denaturation in-solution, judged by the appearance of an ion series representing the apo-protein (heme loss) [17]. $m$-Nitrophenol addition to $5 \mathrm{mM}$ did not affect holo-myoglobin charge state distributions and detached 


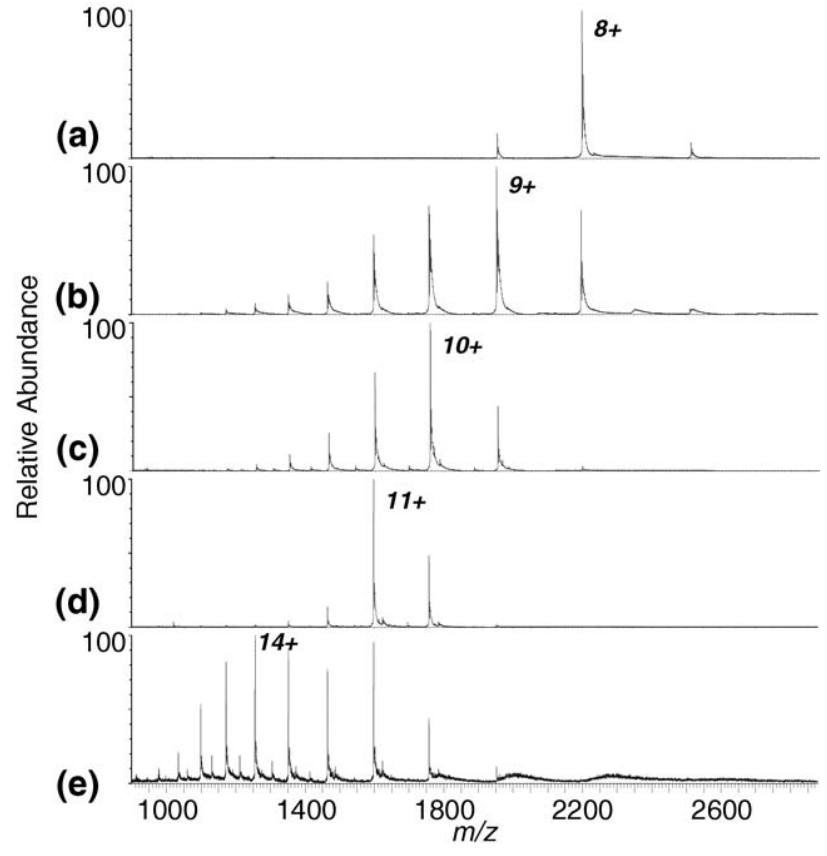

Figure 1. ESI-MS (QTOF/IM) of equine holo-myoglobin in (a) 20 $\mathrm{mM}$ ammonium acetate, and with (b) $1.5 \mathrm{mM} m$-nitrobenzonitrile, (c) $38.8 \mathrm{mM}$ o-NBA, (d) $23 \mathrm{mM} m$-nitrophenyl ethanol, and (e) 276 $\mathrm{mM}$ sulfolane.

no heme. Addition to $20 \mathrm{mM}$, however, induced an $8+-16+$ apo-myoglobin ion series, appearing with relatively unchanged $7+-9+$ holo-myoglobin ions.

Benzyl alcohol is an example of a reagent that increased holo-myoglobin protonation up to a maximum charge of $14+$ in $2.5 \%$ ( $\mathrm{vol} / \mathrm{vol})$, with little evidence of the apo-form, but higher concentrations yielded significant heme loss. The onset of denaturation appeared at about $3 \%$ benzyl alcohol $(\sim 275 \mathrm{mM})$, marked by the appearance of three charge state distributions, two for the holo-protein with maxima at $12+$ and $9+$, and 1 for the apo-form, maximizing at $12+/$ $11+$. Even nitrobenzyl alcohol isomers induced some denaturation; heme loss required $o$-NBA concentrations greater than $38 \mathrm{mM}$. However, even at this concentration apo-ions accounted for only $5 \%$ of the total myoglobin signal.

Increasing the charge deposited on peptides and proteins electrosprayed from denaturing solutions is desirable for many analytical applications. Fortunately, the new reagents also increased protein charge from denaturing solutions. $m$-Nitrobenzonitrile $(22 \mathrm{mM})$ and sulfolane $(300 \mathrm{mM})$ potently supercharge insulin when sprayed from $0.1 \%$ formic acid $/ 50 \% \mathrm{CH}_{3} \mathrm{CN} / 49.9 \%$ $\mathrm{H}_{2} \mathrm{O}$ (vol/vol/vol), shifting insulin's average charge from 4.2 to 5.4 and 5.7, respectively (data not shown).

Many of the charge-enhancing additives we employed are organic solids, expected to reduce surface tension in aqueous systems [18]. Surfactants are also well-known for their ability to reduce surface tension. Surface tensions decrease with increasing surfactant, up to the critical micelle concentration (CMC). In work with nonionic saccharide detergents [19], famed for their ability to solubilize membrane proteins and complexes without denaturation [20,21], we reported that peptide and protein charge state distributions shifted to higher charge with increasing concentrations of the detergents, such as $n$-octyl and $n$-dodecyl glucoside, $n$-dodecyl sucrose, and $n$-dodecyl maltoside. Shifts to higher charge were observed for polypeptides electrosprayed from $100 \% \mathrm{H}_{2} \mathrm{O}$ and from $4 \%$ acetic acid $/ 50 \%$ $\mathrm{CH}_{3} \mathrm{CN} / 46 \% \mathrm{H}_{2} \mathrm{O}$ (vol/vol/vol). Despite reduced surface tension, the average charge observed for denatured bovine ubiquitin shifted from 10.9 to $11.3,12.1$, and 12.3 with $0 \%, 0.01 \%, 0.1 \%$, and $1.0 \% \mathrm{wt} / \mathrm{vol} n$-hexyl glucoside (CMC $=7.0 \% \mathrm{wt} / \mathrm{vol})$, respectively.

Preliminary analysis of the data indicates that "active" reagents appear to have low solution-phase basicities and relatively low gas-phase basicities, revealed by the absence of protonated reagent ions in the low $\mathrm{m} / \mathrm{z}$ region of the spectrum. Both "active" and inactive reagents are less volatile than water. Active reagent ions were observed as clusters, typically charged by addition of ammonium or sodium cations. Not all low-basicity reagents supercharged proteins sprayed from aqueous solutions, however, suggesting that it is only one component of the overall mechanism.

Another feature of mass spectra from some active reagents is that adducts are present on higher charge states (e.g., m-NBA; see Supplemental Materials found in [7]), contrasting with typically observed counterions (e.g., phosphate or sulfate) associated with low charge states. Because ion activation scales with charge, the adducts' association with higher charge states does not reflect mere clustering or incomplete desolvation, processes which would favor lower charge states. NBA's association with low $\mathrm{m} / \mathrm{z}$ myoglobin ions suggests that the mechanism by which proteins acquire additional charge involves direct interaction with the reagent.

Very likely, other factors [22] contribute to the enhanced charging promoted by these reagents. Surface tension $[5,6]$ and solution phase denaturation due to droplet heating [23] are among those that may also play a role. Whether the structure of the macromolecule or charge-enhanced ion has been altered is not clear, and is a question we are actively pursuing. It is clear, however, that these new reagents increase protein charge significantly in ESI of denaturing and nondenaturing solutions, beyond CRM predictions. Maximally 10.4 charges are predicted by CRM for myoglobin sprayed from $\mathrm{H}_{2} \mathrm{O}$ [8], with 8.7, 7.7, and 7.3 charges for solutions supplemented with $m$-NBA, benzyl alcohol, or sulfolane (assuming aged droplets comprised only of supplement molecules). Introducing noncovalent protein complexes with higher charge densities will relax $\mathrm{m} / \mathrm{z}$ requirements for MS detectors and will enhance dissociation efficiency for MS/MS of proteins and complexes. 


\section{Acknowledgments}

J.A.L. acknowledges support from the National Institutes of Health (RR 20004) and the NIH/NCRR High-End Instrumentation Program (S10 RR023045). The authors are grateful for helpful discussions with Jack Beauchamp (Cal Tech), Julie Leary (University of California, Davis), Evan Williams (University of California, Berkeley), and especially Frank Turecek (University of Washington), who suggested sulfolane as a reagent. S.H.L. was supported by the NIH/NIGMS Ruth L. Kirschstein Individual NRSA Fellowship Program (F31GM075384).

\section{Appendix A Supplementary material}

Supplementary material associated with this article may be found in the online version at doi:10.1016/ j.jasms.2009.09.014.

\section{References}

1. Mann, M.; Meng, C. K.; Fenn, J. B. Interpreting Mass Spectra of Multiply Charged Ions. Anal. Chem. 1989, 61, 1702-1708.

2. Loo, J. A.; Edmonds, C. G.; Smith, R. D. Primary Sequence Information from Intact Proteins by Electrospray Ionization Tandem Mass Spectrometry. Science 1990, 248, 201-204

3. Loo, J. A.; Edmonds, C. G.; Udseth, H. R.; Smith, R. D. Collisional Activation and Dissociation of Large Multiply Charged Proteins Produced by Electrospray Ionization. Anal. Chim. Acta 1990, 241, 167-173.

4. Loo, J. A.; Udseth, H. R.; Smith, R. D. Solvent Effects on the Charge Distribution Observed with Electrospray Ionization-Mass Spectrometry of Large Molecules. Biomed. Environ. Mass Spectrom. 1988, 17, 411-414.

5. Iavarone, A. T.; Jurchen, J. C.; Williams, E. R. Supercharged Protein and Peptide Ions Formed by Electrospray Ionization. Anal. Chem. 2001, 73, 1455-1460.

6. Iavarone, A. T.; Williams, E. R. Mechanism of Charging and SuperCharging Molecules in Electrospray Ionization. J. Am. Chem. Soc. 2003, 125, 2319-2327.

7. Lomeli, S. H.; Yin, S.; Ogorzalek Loo, R. R.; Loo, J. A. Increasing Charge While Preserving Noncovalent Protein Complexes for ESI-MS. J. Am. Soc. Mass Spectrom. 2009, 20, 593-596.
8. de la Mora, J. F. Electrospray Ionization of Large Multiply Charged Species Proceeds via Dole's Charged Residue Mechanism. Anal. Chim. Acta 2000, 406, 93-104

9. Šamalikova, M.; Grandori, R. Testing the Role of Solvent Surface Tension in Protein Ionization by Electrospray. J. Mass Spectrom. 2005, 40, 503-510.

10. Iavarone, A. T.; Williams, E. R. Supercharging in Electrospray Ionization: Effects on Signal and Charge. Int. J. Mass Spectrom. 2002, 219, 63-72.

11. Wohlfarth, C. Surface Tension of Pure Liquids and Binary Liquid Mixtures. In Landolt-Börnstein-Group Iv Physical Chemistry Vol. XXIV; Lechner, M. D., Ed.; Springer: Berlin, 2008.

12. Pettit, G. R.; Holzapfel, C. W.; Cragg, G. M.; Herald, C. L.; Williams, P. Broad Scope Secondary Ion Mass Spectrometry. J. Nat. Prod. 1983, 46, 917-922.

13. Rinehart, K. L. Fast Atom Bombardment Mass Spectrometry. Science 1982, 218, 254-260.

14. Jackson, S. N.; Murray, K. K. Infrared Matrix-Assisted Laser Desorption/ Ionization of Polycyclic Aromatic Hydrocarbons with a Sulfolane Matrix. Rapid Commun. Mass Spectrom. 2001, 15, 1448-1452.

15. Meili, J.; Seibl, J. A New Versatile Matrix for Fast Atom Bombardment Analysis. Org. Mass Spectrom. 1984, 19, 581-582.

16. Karas, M.; Bachmann, D.; Bahr, U.; Hillenkamp, F. Matrix-Assisted Ultraviolet Laser Desorption of Nonvolatile Compounds. Int. J. Mass Spectrom. Ion Processes 1987, 78, 53-68.

17. Loo, J. A.; Giordani, A. B.; Muenster, H. Observation of Intact (HemeBound) Myoglobin by Electrospray Ionization on a Double Focusing Mass Spectrometer. Rapid Commun. Mass Spectrom. 1993, 7, 186-189.

18. Holmberg, K.; Jönsson, B.; Kronberg, B.; Lindman, B., Eds. Surfactants and Polymers in Aqueous Solutions, 2nd ed.; Wiley: Chichester, England, 2003.

19. Ogorzalek Loo, R. R.; Dales, N.; Andrews, P. C. The Effect of Detergents on Proteins Analyzed by Electrospray Ionization. In Methods in Molecular Biology: Protein and Peptide Analysis by Mass Spectrometry, Vol. LXI Chapman, J. R., Ed.; Humana Press: Totowa, NJ, 1996; pp 141-160.

20. Barrera, N. P.; Di Bartolo, N.; Booth, P. J.; Robinson, C. V. Micelles Protect Membrane Complexes from Solution to Vacuum. Science 2008, 321, 243-250.

21. Ogorzalek Loo, R. R.; Dales, N.; Andrews, P. C. Surfactant Effects on Protein Structure Examined by Electrospray Ionization Mass Spectrometry. Protein Sci. 1994, 3, 1975-1983.

22. Li, Y.; Cole, R. B. Shifts in Peptide and Protein Charge State Distributions with Varying Spray Tip Orifice Diameter in Nanoelectrospray Fourier Transform Ion Cyclotron Resonance Mass Spectrometry. Anal. Chem. 2003, 75, 5739-5746.

23. Sterling, H. J.; Williams, E. R. Origin of Supercharging in Electrospray Ionization of Noncovalent Complexes from Aqueous Solution. J. Am. Soc. Mass Spectrom., in press. 